\title{
Pour une véritable éducation esthétique
}

\section{Alain Troyas}

\section{OpenEdition}

\section{Journals}

Édition électronique

URL : http://journals.openedition.org/trema/2412

DOI : 10.4000/trema.2412

ISSN : 2107-0997

\section{Éditeur}

Faculté d'Éducation de l'université de Montpellier

\section{Édition imprimée}

Date de publication : 1 décembre 1992

Pagination : 59-77

ISSN : 1167-315X

\section{Référence électronique}

Alain Troyas, « Pour une véritable éducation esthétique », Tréma [En ligne], 2 | 1992, mis en ligne le 01 décembre 1992, consulté le 19 avril 2019. URL : http://journals.openedition.org/trema/2412 ; DOI : 10.4000/trema.2412

Ce document a été généré automatiquement le 19 avril 2019

Trema 


\title{
Pour une véritable éducation esthétique
}

\author{
Alain Troyas
}

\section{1 - Se libérer des dogmes}

1 «Imaginons ». Imaginons le jour où il y aura adéquation entre l'E.A.P. ${ }^{1}$ et une véritable formation esthétique du futur citoyen.

2 Fondons maintenant un espoir. Qu'une éducation artistique ne se soumette à un quelconque dogme aussi établi soit-il et d'aussi haut vienne $t$-il, et qu'elle se conçoive exclusivement sur la base des intérêts des futurs citoyens que sont les élèves.

3 C'est dans cette perspective qui se veut différente de ce que préconise l'actuel programme officiel d'arts plastiques que se présente ce texte. Par ailleurs, il se peut qu'à la lecture de ces lignes on n'y voit rien de contradictoire à l'activité des uns ou des autres. C'est certainement le cas. Il se peut en effet qu'on théorise par ci, par là, et de temps à autre, ou même qu'on dessine parfois encore. La seule différence que je revendique entre ces démarches et ma position, c'est qu'habituellement les propositions présentées ici et qu'on peut en effet retrouver ailleurs ne relèvent pas de ce qu'on appelle une communication incidente (qui fait qu'on peut ou non les rencontrer au gré de possibles aventures pédagogiques), mais qu'elles relèvent d'une communication dite fonctionnelle, autrement dit d'un projet réfléchi et systématiquement appliqué qui fait qu'on ne peut pas ne pas les rencontrer.

4 Ces propositions, à mon sens, tous les élèves devraient pouvoir les rencontrer toutes, et ceci avant leur sortie du système éducatif. Ceci dit, on ne me suivra donc qu'en connaissance de cause. Les propos qui suivent, comme on dit, n'engagent que moi. 


\section{2 - Préparer les conditions d'accès esthétique et non pas enseigner l'art}

5 Car enfin, quel est l'objet d'un enseignement d'arts plastiques? Et que veut-on dire par «accès au monde de l'Art»? Ces questions, il faut en être convaincu, sont déterminantes; de leurs réponses dépend la configuration du terrain à parcourir.

Confrontons-nous donc sans hésitation à ce préalable et tentons d'ôter toute ambiguïté à la fonction d'une éducation artistique « visuelle ». Précisons tout d'abord qu'elle ne doit rien à une quelconque "idéologie charismatique $»^{2}$. A rebours des lieux communs qui courent, il ne peut être question de compter sur une éventuelle révélation miraculeuse qui convertirait l'élève aux mystères de l'Art et qui surviendrait dans le courant d'une aventure que « l'enseignant » ferait vivre à l'élève. Bien au contraire, il faut sans relâche le réaffirmer, l'enseignement est une préparation méthodique qui ne devrait devoir qu'au hasard le tribut d'une découverte impromptue qui est heureusement toujours inévitable en Arts Plastiques. Ici, ce plaisir de la découverte ne peut être que la prime qui distingue ce champ des autres disciplines et non pas le principe directeur de l'enseignement proprement dit. Ne confondons pas "animation culturelle» et enseignement. En conséquence, venons-en aux principes fondamentaux : s'il est donc bien entendu qu'un jour ou l'autre, on ne naît pas sensible au monde de l'art, et aux phénomènes esthétiques en particulier, on conviendra alors que «le talent se forme, l'inspiration s'acquiert, l'émotion se prépare $»^{3}$. L'accès à l'émotion esthétique en effet, se prépare de longue main et à ce titre l'éducation artistique doit être médiatrice vers cette détermination principale qu'est le sentiment esthétique. Il faut préparer le terrain. C'est ce qui importe et à l'encontre de toutes les théories de la révélation, j'avancerai que le territoire de l'EAP ne devrait pas être autre chose que celui où se préparent les conditions d'accès aux phénomènes esthétiques. Rien de plus, mais au-delà de son aspect injonctif, il faut bien mesurer la modestie de ce propos car c'est, à mes yeux, seulement en l'acceptant que peut se fonder la possibilité d'une véritable éducation artistique et éviter les pièges où tombent les pédagogies qui se trompent d'objet. Toutes les pédagogies qui ont méprisé cette humilité et qui ont eu l'ambition déraisonnable d'Apprendre l'art aux élèves, ont connu ou engendreront l'échec. On ne peut en effet apprendre l'Art, cette activité finale qui résulte de la conjonction irrationnelle de facteurs empiriques. En revanche et ceci est le seul espoir de l'existence d'un Enseignement des Arts Plastiques, la seule action possible et même nécessaire d'un enseignant d'Arts Plastiques, c'est de permettre la maîtrise progressive et rationnelle d'outils de connaissance et de stimulation esthétique favorisant l'accès au monde de l'Art et en général au monde des images. C'est à cette action que doit se vouer l'EAP. Je le répète: son territoire est celui de la sensibilité esthétique dans son ensemble indissociable et non par l'une ou l'autre de ses formes particulières telles que l'expression.

\section{3 - Les points cardinaux possibles d'un curriculum conçu comme une carte d'orientation}

7 Ainsi globalement nommé, le territoire de l'EAP demande maintenant à être parcouru en s'orientant selon ses repères naturels qu'il nous reste à découvrir. Il est déjà possible de 
prudemment considérer que toute éducation artistique « visuelle » doit d'abord obéir ici comme ailleurs, à des principes qu'on nomme habituellement en Didactique, des buts et qui servent à désigner les caractéristiques du profil de sortie que la collectivité espère de ses futurs citoyens. On voit ici que cette attitude est également fondamentale. C'est en effet le profil de sortie qui commande les démarches et les objectifs particuliers de tout Programme. Ceci dit, osons une métaphore. Même si la signification du mot «but » est assez transparente, préférons lui pourtant la formule plus imagée de «points cardinaux ». Tout en évoquant encore l'idée des caractères du profil de sortie elle a l'avantage supplémentaire de contenir celle de repères propres à l'orientation et cette précision sera précieuse dans notre périple à l'intérieur de la région à explorer. Ces points cardinaux constitueront tout à la fois les horizons à poursuivre et les limites du territoire de la Sensibilité Esthétique.

\section{Quels points cardinaux?}

8 Si à mon avis, le libéralisme pédagogique et la prééminence de la Pratique n'ont pas à se fonder en tant que point de départ et horizon de la construction d'un Programme, sur quels points cardinaux précis et nommables faut-il alors s'orienter? Evidemment sur aucun principe non justifié par les exigences de la réalité qu'aura à vivre le futur citoyen qu'est l'élève. Ici encore, pas de dogmes mais une orientation fondée sur la question de savoir ce qu'on attend de l'élève à la sortie du système enseignant. Pour nous guider dans la conception de notre tâche convoquons les références habituelles. Construire un programme nous apprend Louis d'Hainaut, c'est répondre aux exigences de ce que la recherche pédagogique anglo-saxonne appelle un «curriculum »4. Nous conviendrons ici que ce n'est que sur cette base qu'une nouvelle carte de l'éducation artistique, organisée et précise, pourra éviter de se contenter d'évoquer les résultats attendus en termes généraux et vagues comme le fait le Programme actuel. Comme le suggèrent les divers auteurs s'en préoccupant, et ceci suggère l'existence de certains de nos points cardinaux recherchés, la conception d'un curriculum a tout intérêt à s'appuyer sur : une description des besoins des élèves et de celles des attentes de la collectivité

9 Les attentes de l'élève : expression et contemplation. S'il est louable, comme le fait l'actuel programme, d'espérer des élèves qu'ils atteignent tous le plaisir d'« accéder au monde de la création» (sous-entendu: de la création expressive instrumentale), cette attente est néanmoins utopique et ne peut qu'engager les pratiques éducatives sur de fausses pistes.

10 Faisons la part des choses. Ce qui est réaliste, c'est d'abord de faire le constat que, dans les classes, tous les élèves n'ont pas les mêmes dispositions. Les uns ont certes le goût de l'expression (la réalisation de dessins, peintures, sculptures, etc.) et s'y adonnent volontiers, mais il est en même temps impossible de ne pas reconnaître que les autres n'en éprouvent pas suffisamment le penchant pour espérer, dans le cadre du faible horaire imparti à l'EAP, avoir l'occasion de rencontrer ce goût et de s'y éveiller. Néanmoins, s'il est déraisonnable d'espérer les voir accéder à ce monde de la Création tel que l'invoquent les Textes officiels, il faut quand même affirmer ici que cette non attirance des seconds pour les activités pratiques ne signifie pas pour autant leur désintérêt pour le monde des images. Car combien parmi ces élèves non motivés par l'expression, peuvent quand même prendre plaisir à la contemplation, cet autre versant de l'activité esthétique? Risquons même une réponse : certainement la grande majorité. 
Ne faut-il pas alors satisfaire cette demande ? A ceux-là ne faut-il pas un enseignement adapté ? Est-il juste et défendable de les contraindre à se livrer exclusivement à des activités d'expression vers lesquelles ils ne sont pas portés ? Est-il juste et défendable de les mettre ainsi en situation d'échec donc de rejet des phénomènes esthétiques? Soyons réalistes et concédons qu'on ne saurait aborder ces deux catégories d'élèves de la même manière. Je proposerai de considérer la formation artistique dans le second degré selon cette perspective : si tous les élèves n'auront pas à se livrer un jour aux plaisirs de la création personnelle, tous gagneront néanmoins à avoir été culturellement préparés aux plaisirs de la contemplation et de la participation aux décisions esthétiques. A ceux qui ont le goût de la pratique, donnons le goût du jugement. A ceux qui n'éprouvent que le besoin de contemplation, donnons-leur, certes, le goût de l'expression mais donnons, leur aussi les moyens du jugement. Ces considérations sont fondamentales. C'est la réalité humaine de laquelle toute orientation pédagogique doit aussi partir.

Concevoir une éducation artistique pour tous, c'est reconnaître qu'on puisse parvenir à cette sensibilité tantôt par l'information conceptuelle selon des dosages à moduler, tantôt par des expériences d'expression pratique. Concevoir un curriculum de l'EAP, c'est distinguer deux voies d'accès dans le monde des images. Il faut accepter de rééquilibrer les cours d'AP aujourd'hui dominés par les activités d'expression conçues comme une voie royale vers la sensibilité artistique et donner une part équivalente aux activités de réflexion et nous tiendrons ainsi nos deux premiers points cardinaux justifiés par l'attente initiale de deux catégories d'individus éprouvant un désir plus ou moins grand soit pour l'expression (le plaisir de réaliser), soit pour la contemplation (le plaisir de regarder) $)^{5}$.

Les attentes de la collectivité : la participation socio-esthétique.

Mais qu'on ne s'y trompe pas. Reconnaitre la réalité de ces deux publics aux sensibilités différentes, ne doit pas conduire pour autant à priver d'éducation artistique visuelle les élèves non motivés par l'expression. C'est s'enfermer dans un piège que de voir dans celle-ci la conséquence de celle-là alors qu'en réalité elles sont l'une et l'autre les deux faces d'une même pièce.

Contre cette façon de voir, il vaut mieux poser le problème autrement: Quelles sont les aptitudes que la collectivité attend de ses membres? Avançons une réponse : sans doute qu'ils possèdent et manifestent tous une sensibilité artistique. Disons le aussi en termes de "profil de sortie », (par la même occasion nous dégageons maintenant les troisième et quatrième points cardinaux qui nous guideront): les attitudes fondamentales que la collectivité attend de ceux qui ont reçu une éducation artistique ne sont pas uniquement de l'ordre de l'expression instrumentale mais elles relèvent aussi et autant de la réception et du jugement. Outre que ce jugement est nécessaire, comme nous l'avons vu, aux plaisirs de la contemplation individuelle, il est également une aptitude précieuse pour la vitalité et la qualité de vie de la collectivité. La création et l'esthétique du monde ne sont pas seulement l'affaire de ceux qui produisent. Il y a aussi et peut-être même d'abord parce qu'ils sont l'immense majorité, ceux qui reçoivent. Ceux-ci ont la charge, (et doivent être éduqués afin d'en avoir envie), de participer aux décisions de politique socioesthétique, c'est-à-dire agir et peser sur les choix configurant l'environnement de tous. Le curriculum d'une véritable éducation artistique doit donc concerner tous ceux qui sont aujourd'hui les usagers du système scolaire et qui seront les futurs citoyens. La collectivité ne pourra alors que bénéficier de la participation consciente et réfléchie de tous ses agents à la création du cadre de vie. L'éducation artistique visant à sensibiliser 
les élèves doit considérer d'abord qu'une totalité sociale est certes composée d'individus pouvant ou non se livrer à une pratique artistique mais surtout devant tous posséder un tronc commun de références permettant des choix culturels et les impliquer ainsi dans l'Imaginaire de leur époque.

Il faut affirmer clairement que l'essentiel est l'Education Artistique Globale, que le monde est composé de producteurs et d'usagers et qu'il s'agit avant tout de les éduquer en vue d'une symbiose de leurs aspirations mutuelles. Dans ces conditions et si on accepte le principe de ce "profil de sortie ", une des priorités d'une véritable éducation artistique n'est pas seulement de former des producteurs d'images mais aussi et même peut-être par dessus tout, des individus capables de vivre plus profondément leur existence en agissant de près et de loin sur les phénomènes qui modèlent leur comportement et leur imaginaire. La collectivité attend de ses citoyens qu'ils participent à l'esprit de leur époque et qu'ils usent pour ce faire des acquis issus de leur formation.

\section{4 - Le principe du curriculum : une carte d'itinéraires}

17 A ce point de mon propos, il me faut rassurer d'avance ceux qui s'effraient de la simple idée de Programme. Un curriculum n'est pas fatalement quadrillé comme un règlement disciplinaire. Ici au contraire, devant nous, le territoire à explorer, même s'il n'est pas vierge, a quand même suffisamment tendance à fréquemment retourner à l'état sauvage pour qu'il laisse aussi à ceux qui le veulent, le pouvoir d'y tracer de nouvelles pistes personnalisées au gré de leur subjectivité et des circonstances. L'essentiel, et j'invite tous mes collègues à réfléchir objectivement à cette question, n'est-il pas de reconnaître que si l'on peut accéder aux centres vivants du territoire de la sensibilité artistique par les chemins les plus variés, certains d'entre eux peuvent aussi se révéler sûrs et surtout indispensables? Si l'on considère d'autre part, comme il sera nécessaire de le faire, l'existence de certains centres vitaux, n'est-il pas élémentaire d'offrir à tous les usagers des itinéraires balisés, des issues de secours? Libres alors aux intrépides de tracer leurs propres pistes, d'escalader leur versant inconnu, pourvu qu'on les retrouve tôt ou tard sur les acquis-refuges communs minimum des élèves... N'est-il pas évident que la collectivité doive imposer aux éducateurs esthétiques qu'ils permettent aux élèves d'utiliser avec pertinence les notions esthétiques de Beau ou de Laid, pour ne prendre en exemple que les plus courantes et les plus galvaudées? Il faut être conséquent: dire qu'un curriculum est contraire aux intérêts de l'EAP et qu'il ne faut pas d'acquis communs précis et nommables, c'est dire que tout se vaut, qu'il n'y a pas de différence de valeur entre, par exemple, l'expression et le jugement, ou encore qu'il n'y a pas plus d'intérêt à s'attarder sur des concepts tels que le Beau que sur la notion de «craie écrasée ». Le curriculum souhaitable doit prendre des responsabilités avec courage. Il existe un certain nombre d'acquis obligés constituant pour tous les élèves le tronc commun de connaissances qui autorise la communication sociale. Ce serait également une erreur de continuer à prétendre que la région où s'épanouit l'Art étant inconnaissable, il ne peut y avoir d'autres avancées que celles du hasard. Il est vrai qu'aux regards les plus initiés comme à ceux qui le sont le moins, le paysage des AP apparaît en effet complexe et changeant. La topographie y est instable, la flore y est vivace. Bien qu'explorées, certaines zones ont des limites incertaines. On le sait suffisamment pour ne pas insister, ce territoire enchevêtré est confondant. 


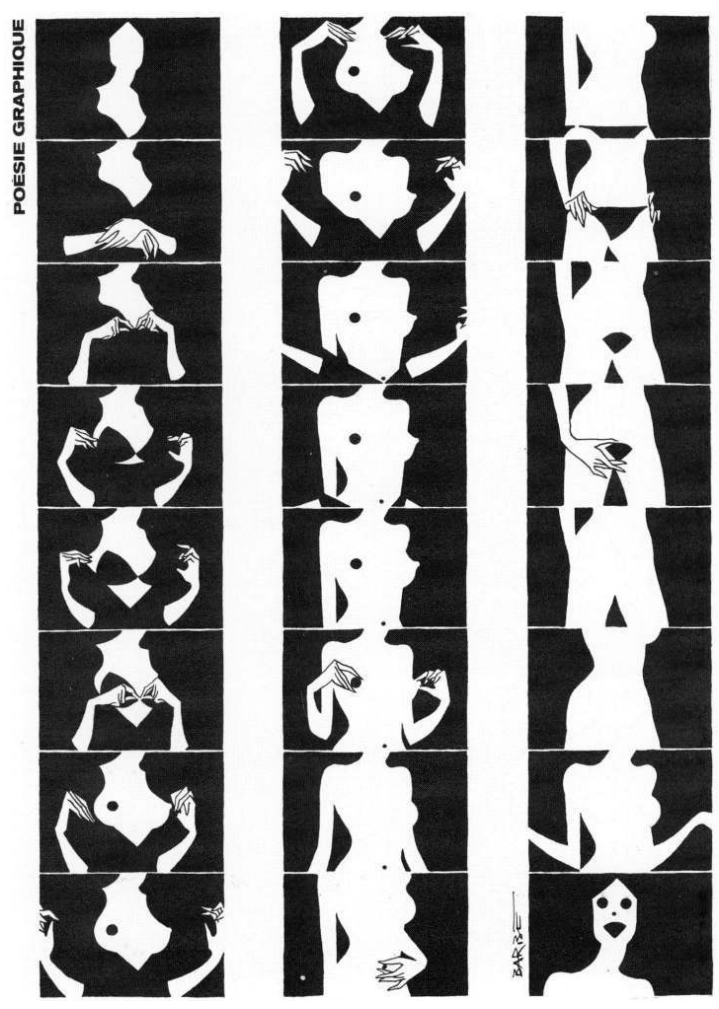

18 Cependant on se méprendrait en prétendant qu'on s'avance là en totale terra incognita. Le champ des images artistiques et communicationnelles est déjà repéré et cette reconnaissance permet de supposer qu'il y a de l'enseignable. Il faut accepter le fait que cet espace soit jalonné de concepts admis sinon vrais, sillonné de trajets culturels valides sinon infaillibles ou bien encore irrigué de flux répertoriés d'habitudes et de comportements stéréotypés à franchir et à dépasser. Ce sont là des parcours et des jalons d'une indéniable utilité qui fonctionnent là encore à la manière de la signalétique des cartes touristiques. Le territoire des AP doit être parcouru comme on parcourt un continent. Bref, un «curriculum » devrait être l'équivalent d'une carte proposant des itinéraires dans un territoire où certaines zones stables en côtoient d'autres qui sont encore en permanente évolution géologique.

19 Simplement, toute l'attention doit être portée sur la souplesse de ses possibilités : la carte doit veiller à offrir à la fois des itinéraires incontournables et des lieux-refuges mais aussi sous forme optionnelle, d'autres chemins, d'autres sentiers, d'autres campements toujours à tracer et à ouvrir. D'abord incontournables. A l'instar des réalités géohistoriques, certains lieux ont une importance centrale qu'on ne peut méconnaître ou éviter. Que penser par exemple, d'un parcours italien qui ignorerait Florence, Rome, Venise? Certainement qu'il gâcherait irrémédiablement la possibilité d'une sensibilisation profonde et vivante. Il en est de même pour l'aventure en territoire artistique. Certains concepts historiques, certains apprentissages techniques, certaines expériences émotionnelles sont les passages obligés de tous les itinéraires. Les éviter ou les ignorer ne peut revenir qu'à s'égarer dans la nuit, à dériver dans l'insignifiance, à s'échouer sur l'accessoire. Les signaler sur la carte programmatique, n'est-ce pas au contraire élever des phares pour accéder plus sûrement au cœur poétique des événements artistiques et n'est-ce pas incomparablement fertiliser les plaisirs d'autres découvertes surprenantes en d'autres chefs-lieux? 
20 L'essentiel est que l'ensemble du public scolaire puisse découvrir dans l'E.A.P. un choix minimum de voies d'accès initiatrices qui le mèneront avec des garanties (mais certes pas en toute sûreté) aux centres culturels les plus panoramiques, les plus animés et les plus vivants, comme autant d'étapes initiatrices vers des destinations capitales. Enfin, un dernier atout : les parcours proposés par un Programme doivent pouvoir servir de guide. Un guide qui permet à tous ses usagers, de contrôler à tout moment si les voies et les étapes proposées conduisent bien à leur enrichissement culturel.

\section{5 - Les régions de l'EAP}

\section{Toutes les images mais seulement les structures communes}

21 Toutes les images. Mais qu'entendre par "accès au monde des images »? Tout simplement que l'élève vivant dans le monde réel, un monde multiple et complexe doit être préparé pour y jouer un rôle et non pas pour le subir. L'éducation artistique des AP doit être complète. L'élève est doté d'une personnalité indivisible. Il devra jouer, en tant que membre d'une famille, d'une communauté ou d'une société, un rôle de participant à la vie culturelle de son temps. L'image sous toutes ses formes sollicite inévitablement cette dimension. Les images utilitaires de publicité, de reportage, d'information, de propagande, et évidemment les images plus purement artistiques (peinture, sculpture, architecture, cinéma), composent l'environnement influençant le comportement. Face à ces deux catégories d'images, l'éducation esthétique ne peut choisir. Elle doit considérer toutes les images. Autant les unes que les autres, elles envahissent le monde, elles déclenchent des modes et orientent spectaculairement ou insidieusement la sensibilité contemporaine. Bien qu'occupant deux régions différentes, elles appartiennent quand même toutes deux au même continent. La carte du monde des images peut donc être délimitée en deux territoires aux frontières naturellement floues mais indiquant cependant une transition entre des types distincts. L'un dont le centre est constitué des images de communication drainées de rationnel et de logique ; l'autre plus symbolique et plus touffu, foisonne de mystères à toujours déchiffrer.

L'essentiel des images. Souple et diversifiée comme l'est le monde, rayonnante dans tous les domaines des phénomènes visuels, l'éducation artistique ne doit pas être seulement complète mais elle doit être également synthétique. Elle doit permettre de saisir, à travers l'abondante incohérence des événements et la diversité des provinces qui la constitue, la profonde unité du continent où elle règne. La difficulté qui paraît insurmontable à beaucoup est celle de l'immensité du terrain et de la variété complexe des événements qui s'y déroulent. Arbre qui cache la forêt, cette complexité a engendré une réponse erronée : un découpage en frontières artificielles, la peinture, le cinéma, la photo, le théâtre... Par ce découpage, on renonce alors à assumer la tâche essentielle de délivrer une culture générale qui seule peut pourtant permettre une compréhension de cette complexité. On renonce donc à découvrir l'Unité de cette diversité et on préfère la découper en pays étanches et fallacieusement autonomes. A l'encontre de ces choix institutionnels qui subsistent encore, affirmons en passant, qu'une éducation artistique complète ne passe pas forcément et toujours par un dispositif optionnel. S'il est vrai que le champ des AP est d'une configuration complexe, il est faux cependant de prétendre qu'à cette complexité on ne peut que donner des réponses compliquées. Plutôt que de séparer en provinces autonomes, la photo du cinéma, la publicité de la peinture, 
l'information visuelle de la suggestion de l'œuvre d'art, plutôt que de se compartimenter, de s'isoler et de se spécialiser, l'EAP dans le secondaire, doit au contraire permettre d'avoir préalablement une vue d'ensemble en sillonnant ces territoires par des artères communes et qui les font vivre comme une totalité organique. Une des taches des promoteurs d'un futur curriculum, sera certainement de trouver et d'entretenir ces organes communs, ces principes généraux qui animent le corps organique du continent.

Dans le second degré, l'éducation artistique comme toutes les autres disciplines ne devrait pas avoir à spécialiser. Elle devrait fournir le corpus commun d'une culture générale et non pas une accumulation d'options illusoirement indépendantes. Au savoir éclaté et incohérent, l'école doit opposer le savoir organisé 6 . Au collège et au lycée, il ne peut y avoir d'un côté, un enseignement des images de communication, et de l'autre un enseignement des images artistiques. Toutes les images participent de l'E.A.P. De cette unité doivent apparaître des structures communes essentielles faisant l'objet d'un enseignement commun pour tous.

\section{L'imaginaire}

Vers où avancer dans ces régions à la topographie et à la flore si variées? Quels havres, quels objectifs doivent servir de relais pour tout à loisir pouvoir à la fois décrypter et déjouer, et aussi contempler et fabriquer?

Si jouer un rôle social culturel, c'est décrypter autant que fabriquer ces images qui façonnent la personnalité, toute éducation artistique doit alors examiner les courants internes qui partagent le territoire des images selon deux points de vue : dans la région "Communication " selon un point de vue défensif contre leurs effets manipulatoires du comportement et surtout dans la région «Symbolique » selon un autre point de vue, plus créatif celui-là, pour en assimiler ainsi que l'a vu Bruno Duborgel ${ }^{7}$, les contenus imaginaires et poétiques enracinés dans le tissu social qui nourrissent et fécondent les propensions naturelles de tous à la fiction. L'EAP doit se concevoir comme une culture systématique et comme un développement du réservoir mental alimentant l'imaginaire de tous les élèves quelles que soient leurs dispositions. Un certain nombre de mythes, parmi les plus importants et les plus structuraux de notre environnement culturel pourraient être inscrits dans le patrimoine à visiter. Ainsi focalisé, (au sens où l'entend d'Hainaut pour désigner sa programmation) par rapport aux intérêts des individus et de la société, un curriculum artistique dans le second degré aurait à tenir compte de la totalité du potentiel culturel des futurs citoyens.

\section{Motiver en allant droit au but : «A quoi servent les AP ? »}

Le corpus d'une véritable éducation artistique devrait donc être synthétique et construit en tant que préparation où se mettent systématiquement en place les conditions d'accès aux phénomènes esthétiques. Il faut alors vaincre le premier et le plus grand obstacle : la faible motivation originelle des élèves pour ces voyages culturels initiatiques. Le moins qu'on puisse dire, c'est qu'à leurs yeux, la détente agréable est la justification majeure de l'E.A.P. Pour eux qui mesurent l'importance d'une discipline à l'aune de son « utilité », la séance d'A.P n'a souvent rien de nécessaire... Le problème est décisif, plus que tout autre il mérite qu'on s'y attarde et il faut se poser la question. Pourquoi les élèves considèrentils l'EAP davantage comme un délassement que comme une activité aussi fondamentale 
que les mathématiques par exemple ? Parce que, je le soutiens, entre toutes les causes de cette désinvolture, la plus importante et la plus grave réside dans l'ignorance de l'importance de sa fonction psycho-sociologique. Alors que les images conditionnent pour ainsi dire le climat sous lequel on vit, peu d'élèves voient la force de leur influence. Encore moins estiment qu'il est important d'en comprendre les conditions de formation. Pour la plupart d'entre eux, ces phénomènes relèvent soit du surnaturel soit de l'évidence et paraissent aussi inaccessibles que les phénomènes météorologiques. Lever les yeux vers ces hauteurs où se forment ces phénomènes, relève alors de leur point de vue, de l'occupation du temps libre, pas de la nécessité. A la limite, s'adonner aux Arts Plastiques est un luxe au même titre qu'un voyage estival. On se dépayse en visitant une lointaine contrée, on en revient avec des souvenirs sans doute, mais on n'y habite pas. Ainsi en estil du monde des images et de l'émotion esthétique. On peut éventuellement contracter un voyage guidé par un quelconque tour operator, mais on ne peut concevoir d'en faire son environnement permanent.

Or, la tâche permanente de l'EAP est de dissiper ce malentendu. Il est absolument vital de convaincre les élèves de l'importance de ce climat esthétique sous lequel ils vivent et par voie de conséquences de la nécessité d'une formation préparatoire. Cette conviction est loin d'être le cas, dans leur conscience comme dans celle de leurs parents sans doute, mais ceci n'est possible que parce qu'avant tout elle est loin d'être présente dans la conscience de l'Institution elle-même. L'adhésion des élèves aux diverses propositions éducatives artistiques devrait donc faire l'objet d'un souci permanent de la part de l'Institution, et ceci pendant toute la scolarité. Il faut les convaincre de l'importance de la fonction des images dans la communauté sociale. Ce n'est qu'à ce prix que le cours d'AP ne sera pas subi comme une contrainte routinière et inévitable, ou encore attendu comme une simple récréation. Il doit être vécu comme un itinéraire initiatique menant à la compréhension du monde et de soi-même. Un curriculum devrait inscrire à chacune de ses pages et en les adaptant à chaque niveau scolaire, les opérations qui sont destinés à convaincre de l'importance des phénomènes esthétiques. Cette conviction ne peut se faire comme c'est actuellement le cas, à travers des manipulations hasardeuses et gratuites, des périphrases incertaines et compliquées, mais en posant le plus directement les questions: quelle est la fonction des images? Comment influencent-elles le fonctionnement des sociétés? Comment se posent-elles en enjeux de civilisation? Comment agissent-elles sur le goût et le comportement individuel ? Il faut se convaincre de l'importance prioritaire de cet impératif. C'est seulement en élargissant à cette dimension initiatrice le champ de conscience des élèves que toutes les autres opérations de développement du comportement artistique ont quelques chances de réussir.

\section{Les lieux sémiotiques de connaissance}

Si la motivation indispensable des élèves passe par une claire conscience des finalités d'une sensibilité esthétique, comme initiation à un monde désirable, elle doit aussi avoir visité des lieux de connaissance qui permettent de confirmer l'intérêt promis de l'itinéraire proposé. La mobilisation de l'intérêt des élèves passe par l'acquisition d'un début de compétence conceptuelle clairement ressentie comme positive grâce à une vérification expérimentale. Les productions à caractère artistique se situant toujours au carrefour de Sciences Humaines, on a tout intérêt à faire appel aux notions que ces sciences ont dégagées. Qu'est-ce que comprendre les œuvres artistiques, les productions 
symboliques, sinon comprendre en quoi elles sont, entre autres, signes, emblèmes ou symboles? N'y a t-il pas dans la simple énonciation de cette structure sémiotique un repère propre à favoriser l'imagination des élèves tout en stimulant la liberté de l'enseignant? Ces concepts sont chargés de sens comme le sont certains monuments qui concentrent en eux les strates des sensibilités historiques. Les visiter c'est rechercher des angles de vue valorisant leurs fonctions expressives et leur retentissement sur les ouvrages environnants. Là aussi, devant ces concepts, l'approche est variable selon les éclairages qu'on leur a donnés, comme peuvent l'être ceux qu'on projette sur une sculpture, une architecture ou un paysage. Mais l'essentiel est d'en avoir saisi l'importance et de s'en approprier les messages comme des clés ouvrant d'autres horizons. Quoi qu'il en soit, les voies d'accès à ces concepts opératoires sont infinies. Les moyens d'y accéder ne peuvent qu'être aussi diversifiés que la perspicacité des enseignants. S'il est prioritaire de permettre la prise de conscience de l'importance de la fonction de l'art sur le comportement collectif et individuel, il est aussi fondamental d'initier les élèves au maniement d'outils intellectuels favorisant la compétence à saisir les distinctions sémantiques fondamentales entre les grandes catégories d'objets esthétiques. En proposant des concepts opératoires à acquérir on conduit une 1ère approche rigoureuse riche d'avenir, d'un vocabulaire structurel de formes et de signification à la portée d'élèves de 6ème comme de 3ème.

\section{Les notions de psychologie}

Si toute image est le produit d'un investissement de sens social que repère entre autres la sémiotique, elle est aussi le produit d'une activité psychologique que tous les élèves gagnent à comprendre et à reconnaître consciemment comme faisant partie intégrante de leur vie mentale et biologique. Comment ignorer en effet, les concepts qu'a dégagés la psychanalyse notamment, pour rendre compte des processus internes et subjectifs souterrains d'où jaillissent incandescentes, les images, les œuvres? Sous cet angle, là aussi, informer de ces réalités conceptuelles c'est du même coup démultiplier les richesses du sens des images. Comme l'effet des volcans, leur impact sur l'imaginaire ne peut que saisir l'esprit. Plus opératoirement et pour prendre un exemple, Freud a présenté le processus du rêve comme une métaphore du travail de création artistique (et inversement). Les sourdes et invisibles opérations intimes constitutives de l'élaboration du rêve que sont le déplacement et la condensation éclairent alors en conséquence, de feux fascinants la production d'images. Ces deux notions ouvrent des portes aux questions que pose le mystère des œuvres. Pourquoi ne pas les impliquer dans un enseignement? Continuons sur cet exemple en ajoutant à ces deux repères conceptuels fondamentaux d'une culture de base, ceux bien sûr des notions d'inconscient et de sublimation. Peut-il y avoir aujourd'hui une compréhension minimum des phénomènes esthétiques sans une reconnaissance de ces principaux concepts psychanalytiques? Sans doute le périple en cette région est-il difficile et dangereux. Ces repères notionnels sont certes d'un abord malaisé tant leur investissement peut perturber certains équilibres. A cette prudence légitime, il faut répondre avec l'argument que j'ai dénié aux partisans d'une vision « animatrice » des Arts Plastiques au collège et au lycée et que je considère comme ne pouvant être que la prime de l'enseignement. L'exploration du monde des Images et le monde de l'Art sont aussi (mais seulement aussi) une aventure. La complexité de la configuration de ces repères fait certes courir des risques à toute entreprise pédagogique, mais l'époque et ses enjeux obligent à les accepter dans ce cas précis des 
problèmes que provoque la psychanalyse. Il faut le répéter, s'il faut sensibiliser les élèves à une future liberté intellectuelle et les aider à construire un comportement esthétique, alors il faut prendre ses responsabilités. Il faut accepter les résultats du travail séculaire de la culture. Il existe des notions-clés dont aucune investigation sérieuse ne peut se passer. Elles ouvrent la compréhension et l'action dans les mondes où nous nous aventurons. Les psychologies et plus particulièrement la psychanalyse, offrent certaines de ces clés. Une compréhension des phénomènes artistiques peut-elle aujourd'hui se passer des concepts qu'ont construits les Psychologies? Il est temps que le système éducatif s'aperçoive qu'aucune politique d'éducation artistique ne peut éviter d'inscrire dans son programme scolaire au collège comme au lycée les questions posées par la psychologie des profondeurs.

\section{Le contenu esthétique}

Evidemment, aucune sensibilisation efficace aux mondes des images artistiques ou utilitaires ne peut ignorer le sens des mots qu'on utilise à tout propos et malheureusement trop souvent hors de propos. Pourquoi par exemple dans le langage courant invoque $t$-on si volontiers des catégories esthétiques telles que le beau, le laid, le poétique, le tragique, etc. pour énoncer encore des jugements de goût? Toute l'histoire du goût de la civilisation occidentale est imprégnée de conceptions esthétiques dont le sens et la valeur méritent une évidente et permanente sollicitude, et ceci dès la 1ére année de collège. Tous nos comportements et attitudes esthétiques tournent autour de ces véritables chefs-lieux conceptuels que sont ces catégories. Tous les édifices intellectuels contemporains qui se dressent aujourd'hui dans les zones de la Critique d'Art n'ont de sens qu'en fonction de ces critères classiques. Comment ne pas les répertorier dans notre utopie? Ce n'est que par une familiarisation raisonnée régulée et spécialisée avec ces concepts, que les élèves accèderont à une intelligence des choix esthétiques qu'ils ont et qu'ils auront constamment à opérer. Comme ailleurs dans les autres secteurs de la connaissance, dégager un corpus léger de concepts théoriques utilisables n'est pas seulement justifié pour répondre à des choix visant l'environnement en général, mais aussi pour porter une appréciation sur le type d'activité à laquelle on s'adonne en tant qu'individu. La culture esthétique n'est pas une afféterie prétentieuse. Elle n'est pas non plus un vernis désuet pas plus qu'elle n'est devenue inopérante. Ses catégories, lentement dégagées par l'Histoire, représentent encore le contenu même de toute attitude socio-artistique, qu'elle soit tournée vers les choix d'orientation culturelle sociale ou qu'elle soit destinée à approfondir la sensibilité de l'individu. A cet égard, les catégories traditionnelles telles que le beau, le laid, le poétique, l'utile, etc. doivent figurer dans l'EAP comme autant de passerelles qu'on ne peut éviter d'emprunter pour parvenir aux destinations souhaitées.

\section{L'histoire de l'art}

Rappelons que rien ne peut se construire sans références communes à une expérience collective, sociale et historique. C'est cette expérience consignée, commentée, sélectionnée par la mémoire historique qu'il faut mettre en perspective devant le regard des élèves. C'est bien là, la fonction de ce qu'on désigne par Histoire de l'art. Or, évoquer cette Histoire de l'Art c'est souvent susciter un sentiment de rejet pour une discipline qu'on peut considérer touffue, scholastique et ingrate. Voici alors un nouveau marécage à 
éviter. Là aussi, le défi est à relever car il est possible de transformer l'insalubrité de cette zone si on accepte d'y planter les fleurs de l'Esthétique. Plutôt que de se présenter comme un morne terrain inorganique, l'Histoire de l'Art doit apparaître comme un jardin où sont mis en valeur les pièces les plus spectaculaires des sensibilités culturelles du passé menant aux emplacements contemporains. Comprendre et agir dans le monde de l'Art contemporain c'est évidemment en situer sa position par rapport à l'Art Moderne et à ses prédécesseurs, ceci est de l'Histoire de l'Art, de la chronologie. Mais cette compréhension espérée est vouée à l'échec si on ne fait appel aux explications de sens dont en général se charge l'Esthétique. Faire par exemple une halte sur les rives de la Figuration Libre oblige à tenir compte du climat général de l'époque qu'est le Post-Modernisme et c'est aussi le mettre en rapport avec la question des Avant-Gardes et ceci n'est éclairant que dans la mesure où on se réfère à la tradition séculaire de la Renaissance. Obéir à ces derniers principes, c'est mêler organiquement l'Histoire de l'Art à l'Esthétique. C'est donner du sens à la chronologie.

On le conçoit alors clairement, le terrain où l'on risquait l'enlisement pour cause d'encyclopédisme, est maintenant devenu attractif parce qu'il concourt à la compréhension du monde, et pas seulement de celui des Images.

Mêler Histoire et Esthétique, c'est aussi impliquer un débat sur les conditions sociales d'apparition des mouvements artistiques (par exemple la question du Réalisme ou de l'Abstraction ne sont compréhensibles qu'en les replaçant dans leurs contextes afin d'en percevoir les effets sur notre sensibilité actuelle). Les contextes de l'œuvre ou de l'image ne peuvent être ignorés. Ils doivent être délimités et inscrits dans le programme comme autant de notions ne se réduisant jamais à une histoire des formes seules. ${ }^{8}$

\section{La pratique}

On le voit assez clairement, du moins je l'espère, ramener une éducation artistique aux activités instrumentales ne répond pas à une véritable éducation artistique. Privilégier la pratique en espérant qu'elle réponde à une attente sociale, est l'erreur paralysante sur laquelle se fonde la vieille idéologie de l'Institution. Une véritable éducation artistique doit poursuivre une finalité clairement démarquée de ce privilège exorbitant accordée à la pratique instrumentale. Réaffirmons le sans faiblesse, la principale finalité qu'on doit attendre d'elle c'est qu'elle cultive avant tout toute la sensibilité esthétique. A cet égard il n'y a pas de raison de donner une quelconque prédominance aux activités instrumentales sur les activités théoriques. Théorie et Pratique doivent être dosées en fonction des impératifs institutionnels qui devraient être conçus comme d'heureux compromis entre la satisfaction de la demande individuelle et l'attente sociale. Mais qu'on ne se méprenne pas, loin de moi l'idée de participer à une entreprise de dévalorisation de la pratique instrumentale. Il reste, et c'est indéniable, que par son caractère à la fois structurant et cathartique, cette activité pratique, concrète, poétique, est un puissant levier pédagogique. Elle est structurante, parce qu'en se livrant à ses opérations, le sujet s'expérimente. Cathartique parce qu'en s'expérimentant concrètement, il se livre aux bénéfices psychiques du jeu. Mais de ce point de vue, là encore, il faut envisager un rééquilibrage du type des activités constituant la pratique dans les AP. Trop souvent dominée par ce qu'il est convenu d'appeler les activités d'Expression, elle néglige les activités d'apprentissage plus rigoureuses telles que le Dessin et ses satellites. Des systèmes optionnels internes au groupe classe doivent être proposés pour valoriser cette 
activité incomparablement efficace pour le développement psychophysiologique des élèves. Disons le encore plus fort et plus clair: il faut revaloriser cette dimension irremplaçable qu'est le Dessin en la réintroduisant royalement et en toute justice dans la carte d'un futur curriculum. Dans cette nouvelle perspective, il ne peut évidemment pas exister d'Education artistique "visuelle» sans qu'on reconnaisse droit de cité à la pratique instrumentale. Mais loin d'être totalitaire, car moyen et fin exclusifs à la fois qu'elle était, elle n'est maintenant qu'une des voies à l'accès esthétique du monde des images. Répétons le: faire aimer l'art, "goûter l'œuvre de Fra Angelico ", ou celle de Schnabel, comprendre et déjouer la publicité, donner envie de peindre ou de faire un film, n'implique pas obligatoirement un parcours uniquement instrumental. Ceci est évident. Mais accéder au contenu de cette mission de l'éducation artistique demande qu'on passe aussi par la pratique instrumentale. Dans le cadre de cette Pratique, un rééquilibrage s'impose entre Expression et Maîtrise Technique. Toute modulation de ces composants de la pratique instrumentale doit accorder une place particulière au Dessin.

\section{6 - Conclusion}

Sans aucun doute, l'actuel programme de l'E.A.P. ne peut servir la fonction d'une véritable éducation artistique tant sa conception est focalisée sur des contenus séparés, décomposés. Il lui manque de tenir compte de la seule dimension réellement unifiante de l'image et de l'œuvre artistique: le sens, cette dimension globale qui condense les multiples influences d'où s'irradient ses significations. Voilà la dimension essentielle manquée par les vieilles orientations. Voilà pointant la seule dimension qui peut créer un Programme authentiquement éducatif. Faire percevoir dans des parcours pédagogiques obligatoires, l'enjeu des œuvres et des images dans le réel et l'imaginaire, donner les moyens d'accès à cette perception, c'est ce à quoi j'invite tous les esprits non dogmatiques. Voilà ce qui devrait être soumis à l'étude d'un nouveau programme. A travers les concepts centraux que cette lointaine vue aérienne a tenté de mettre en relief, une logistique plus sophistiquée doit d'urgence apporter plus de précision pour enfin inaugurer une véritable éducation non pas seulement « artistique », mais aussi avant tout esthétique.

\section{Définitions}

Toutes les définitions ci-dessous sont empruntées à l'ouvrage de Louis D'hainaut: « Des fins aux Objectifs ».

37 Attente : effets espérés d'une formation tels qu'ils sont perçus -mais pas nécessairement exprimés ou entièrement conscients- par les personnes formées ou susceptibles d'entrer dans la formation.

But : résultat attendu d'une formation au niveau de la maitrise d'une situation globale. Ils doivent être exprimés en termes d'activités que l'apprenant devra pouvoir maitriser. Un but est plus général et plus vague qu'un «objectif » mais il est plus spécifique et plus précis qu'une fin, une intention ou une finalité.

Curriculum : plan pour une formation ou une action éducative. Ce plan doit contenir la spécification des résultats attendus de la formation ou de l'action éducative, des moyens prévus pour la réaliser et des moyens envisagés pour en évaluer les effets.

Instrumental : employé ici à propos des pratiques d'outils et des techniques et qui 
servent de moyens pour l'effectuation de savoir-faire.

Profil de sortie : ensemble des savoirs, savoir-faire et savoir être que les personnes formées maitriseront à la sortie de la formation. Il définit les compétences de l'individu formé à l'issue de la formation. Le profil terminal exprimé en termes de capacité de la personne formée remplace les programmes exprimés uniquement en termes de matières.

\section{BIBLIOGRAPHIE}

BOURDIEU Pierre. PASSERON Jean-Claude, L'amour de l'art, Ed. de Minuit, Paris 1969

D'HAINAUT Louis, Des fins aux objectifs, Ed. Nathan, Paris 1985

DOMENACH Jean-Marie, Ce qu'il faut enseigner. Ed. du Seuil, Paris 1990

DUBORGEL Bruno, Imaginaire et pédagogie, Ed. du sourire qui mord, Paris 1983

PORCHER Louis, «Aristocrates et roturiers ». L'éducation esthétique. Ed. Armand Colin, Paris 1973, p. 9.

\section{NOTES}

1. EAP : Enseignement des Arts Plastiques

2. BOURDIEU Pierre, PASSERON Jean-Claude, L'Amour de l'Art, Ed. de Minuit, Paris 1969

3. PORCHER Louis, L'éducation esthétique. “Aristocrates et roturiers”, Ed. Armand Colin, Paris 1973, p.

9

4. D'HAINAUT Louis. Des fins aux objectifs. Ed. Nathan. Paris 1985

5. D'HAINAUT Louis, Des fins aux objectifs, Ed. Nathan, Paris 1985

6. DOMENACH Jean-Marie, Ce qu'il faut enseigner, Ed. du Seuil, Paris 1990

7. DUBORGEL Bruno, Imaginaire et pédagogie. Ed. du sourire qui mord, Paris 1983

8. PANOFSKY Erwin, Essai d'iconologie. Ed. Gallimard, Paris 1979

\section{RÉSUMÉS}

L'actuel programme officiel concernant l'Enseignement des Arts Plastiques est inadapté. Il ne prépare pas tous les élèves à participer à la construction de l'ensemble de l'environnement visuel. Comment y parvenir? Par l'introduction d'un enseignement fondé sur les Sciences humaines. Dans la perspective d'une pédagogie différenciée, un tel parcours s'articulerait certes au monde des images mais aussi autour d'une culture générale où les psychologies autant que les sociologies, l'esthétique ou l'histoire de l'art ne pourraient que fertiliser un terrain trop peu fréquenté. 
Not available

\section{AUTEUR}

ALAIN TROYAS

Professeur agrégé d'Arts plastiques, université Paul Valéry de Montpellier 